\title{
Production of fucoxanthin-rich fraction (FxRF) from a diatom, Chaetoceros calcitrans (Paulsen) Takano 1968
}

\begin{abstract}
Fucoxanthin is the major carotenoid produced by diatoms and shown to exhibit potent antiobesity and anti-cancer activities. This study presents a simple protocol for the preparation of fucoxanthin-rich fraction (FxRF) with high antioxidative properties from a selected marine diatom, Chaetoceros calcitrans. Fucoxanthin concentrates were prepared from crude methanolic extract (CME) with liquidï liquid partitioning of three types of solvent systems (i.e. diethyl etherï water, dichloromethaneï water and ethyl acetateï water), followed by comparative antioxidant evaluation. Dichloromethane fraction (DCMF) was found to contain significantly higher $(\mathrm{p}<0.05)$ level of carotenoid $(7.13 \pm 0.01 \mathrm{mg} \cdot \mathrm{g} \overline{\mathrm{l}} 1 \mathrm{DW})$ and fucoxanthin $(5.25 \pm 0.03 \mathrm{mg} \mathrm{FX} \cdot \mathrm{g} \overline{\mathrm{l}} 1 \mathrm{DW})$ contents accompanied with elevated $(\mathrm{p}<0.05)$ antioxidant activities (DPPHradical dot and ABTSradical dot+ radical scavenging, and beta carotene bleaching assays) as compared to other tested fractions. This study showed that FxRF with enhanced antioxidant properties could be effectively produced and concentrated through the DCMï H2O partition of CME.
\end{abstract}

Keyword: Fucoxanthin-rich fraction; Carotenoids; Microalgae; Chaetoceros calcitrans; Antioxidant activity 